\title{
Percepción del paciente acerca de la calidad de atención odontológica
}

\author{
Perception of the patient about the quality of odontological \\ care
}

\section{Percepção do paciente sobre a qualidade do cuidado dental}

Fabiola Mendoza Morales

fabiolamen2020@gmail.com

Recibido septiembre 2019 / Revisión octubre 2019 / Aceptado 1 de enero 2020

\begin{abstract}
RESUMEN
Introducción: Los pacientes al acudir a un servicio presentan ciertas expectativas en cuanto al servicio recibido. Objetivo: La investigación que se resume en este artículo tiene por objetivo determinar la percepción del paciente acerca de la calidad del servicio y atención odontológica que se le brinda en las clínicas de la Facultad de Odontología de la Universidad de San Francisco Xavier de Chuquisaca. Materiales y métodos: Es un estudio cualitativo, con método etnográfico, mediante el empleo de la observación participante. La técnica de recolección de datos utilizada fue la entrevista semiestructurada con preguntas abiertas $\sin$ un orden preestablecido adquiriendo características de conversación. Las sesiones se transcribieron para facilitar el análisis de la información, utilizando las siguientes dimensiones de atención de calidad: acceso y rapidez, comodidad, señalética, competencia técnica, relaciones interpersonales, continuidad, seguridad, eficacia y eficiencia. Conclusión: La percepción del paciente en relación a la calidad del servicio y atención odontológica que se le brinda en las clínicas, es buena. Se recomienda establecer mecanismos de seguimiento a la calidad de atención recibida y la eficacia de los tratamientos realizados en las Clínicas Odontológicas.
\end{abstract}

Palabras clave: Calidad; atención salud; eficacia; percepción

\begin{abstract}
Introduction: Patients when going to a service have certain expectations regarding the service received. Objective: The research summarized in this article aims to determine the patient's perception of the quality of dental service and care provided in the clinics of the Faculty of Dentistry of the University of San Francisco Xavier de Chuquisaca. Materials and methods: It is a qualitative study, with an ethnographic method, using participant observation. The data collection technique used was the semi-structured interview with open questions without a pre-established order acquiring conversation characteristics. The sessions were transcribed to facilitate the analysis of the information, using the following dimensions of quality care: access and speed, comfort, signage, technical competence, interpersonal relationships, continuity, safety, effectiveness and efficiency. Conclusion: The perception of the patient in relation to the quality of the service and dental care provided in the clinics is good. It is recommended to establish follow-up mechanisms for the quality of care received and the efficacy of the treatments carried out in the Dental Clinics.
\end{abstract}

Key words: Quality; health care; effectiveness; perception

$\begin{array}{lrr}\text { FM: } & \text { Universidad } & \text { San } \\ \text { Francisco } & \text { Xavier } & \text { de } \\ \text { Chuquisaca, } & \text { Bolivia. }\end{array}$




\section{RESUMO}

FM: Universidad San Francisco Xavier de Chuquisaca, Bolivia.
Introdução: Os pacientes quando vão a um serviço têm certas expectativas em relação ao serviço recebido. Objetivo: A pesquisa resumida neste artigo tem como objetivo determinar a percepção do paciente sobre a qualidade do serviço e atendimento odontológico prestado nas clínicas da Faculdade de Odontologia da Universidade de São Francisco Xavier de Chuquisaca. Materiais e métodos: Estudo qualitativo, com método etnográfico, utilizando observação participante. A técnica de coleta de dados utilizada foi a entrevista semiestruturada, com perguntas abertas, sem ordem pré-estabelecida, adquirindo características de conversação. As sessões foram transcritas para facilitar a análise das informações, utilizando as seguintes dimensões do atendimento de qualidade: acesso e rapidez, conforto, sinalização, competência técnica, relacionamento interpessoal, continuidade, segurança, efetividade e eficiência. Conclusão: A percepção do paciente em relação à qualidade do serviço e atendimento odontológico prestado nas clínicas é boa. Recomenda-se estabelecer mecanismos de acompanhamento para a qualidade do atendimento recebido e a eficácia dos tratamentos realizados nas Clínicas Odontológicas.

Palavras-chave: qualidade; assistência médica; eficácia; percepção

\section{INTRODUCCIÓN}

$\mathrm{L}$ a calidad de atención en salud, es una de las mayores preocupaciones de quienes tienen la responsabilidad de la prestación de los servicios de salud y una necesidad de quienes requieren de estos servicios. La perspectiva del paciente es muy importante porque es más probable que los pacientes satisfechos cumplan con el tratamiento y continúen utilizando los servicios de salud. Es por ese motivo que, la satisfacción del usuario afecta la salud y el bienestar de la sociedad, lo que trae como consecuencia el mejoramiento de su calidad de vida. Se debe pensar que antes de interactuar con el paciente es prioritario conocer su contexto económico y social para mejorar uno de sus puntos más sensibles que es la relación odontólogo- paciente.

Para Donabedian (1), la calidad de la atención es aquella que se espera que pueda proporcionar al usuario el máximo y más completo bienestar después de valorar el balance de ganancias y pérdidas que pueden acompañar el proceso en todas sus partes (1).
Actualmente, una institución de salud que esté interesada en garantizar la efectividad de la atención sanitaria y eficiencia en la gestión, debe conocer las percepciones de los pacientes respecto a los procesos asistenciales, por lo que es importante determinar y supervisar parámetros de calidad relacionados con infraestructura, equipamiento, procesos y personal vinculados a la atención de salud prestada, con el fin de lograr la mayor satisfacción de los pacientes.

En Bolivia se han realizado esfuerzos para garantizar la satisfacción del usuario desde 1990, con este objetivo se creó un Proyecto Nacional de Calidad en Salud, con una propuesta de transformación que exigía contemplar aspectos relacionados con la evaluación y garantía de calidad, es así que, en el año 2000, el Instituto Nacional de Seguros de Salud (INASES) implemento instrumentos de control médico en servicios de salud (2).

El Plan Estratégico de Salud 20062010 del Ministerio de Salud de Bolivia, establecía la implementación de la gestión de calidad en las redes de salud en 4 etapas: inicio, desarrollo, consolidación, 
control y seguimiento; sin embargo, pese a los esfuerzos de los entes gestores no se ha completado el proyecto. Al momento se evalúa anualmente la acreditación de los establecimientos de salud, pero existe poca difusión y aplicación de instrumentos y documentos de Gestión de la calidad en Odontología.

La percepción y expectativas de un servicio cambian de generación en generación, aspectos que hacen necesario conocer las tendencias, intereses y necesidades de los usuarios con el propósito de identificar las oportunidades de mejora que se presentan en el proceso de atención.

Los procedimientos odontológicos que se organizan en servicios odontológicos, según la clasificación de Lamata et al (3) éstos comprenden acciones preventivas, curativas y de rehabilitación; a los que se suma la creciente demanda por los procedimientos estomatológicos de carácter puramente estético (aclaramientos, carillas y despigmentaciones). La investigación del consumidor es parte de la investigación del marketing y es definida como un proceso sistemático y objetivo de recolección y análisis de datos relativos al comportamiento del consumidor (4).

Con el presente estudio, se pretendió conocer la percepción que el paciente tiene acerca de la calidad del servicio y atención odontológica que se le brinda en las Clínicas de la Facultad de Odontología de la Universidad de San Francisco Xavier de Chuquisaca. Los resultados obtenidos permitieron establecer una base para mejorar la calidad de atención, con la consecuente mayor satisfacción de los pacientes.

\section{MATERIALES Y MÉTODO}

$\mathrm{E}$ sta investigación siguió lineamientos de tipo cualitativos. La población de estudio fueron tres pacientes por cada especialidad que se atiende en las clínicas de la Facultad de Odontología: Operatoria DentalEndodoncia, Cirugía- Periodoncia, Prótesis Fija-Prótesis Removible, PediatríaOrtodoncia del turno de la mañana y el turno de la tarde, haciendo un total de 48 pacientes.

La técnica de análisis utilizada fue descriptiva o interpretativa, dentro de la cual se aplicó una técnica conversacional para conocer el punto de vista y las experiencias del informante, denominada entrevista semiestructurada. En la entrevista semiestructurada, la conversación se guía en torno a unas cuestiones prefijadas en la que se plantea una secuencia de preguntas abiertas. Se realizó el análisis de contenido, para ello, se segmentó el texto según las dimensiones de calidad de atención en salud investigadas en cada una de las clínicas de la Facultad de Odontología que permitieron describir lo tratado.

Para determinar la percepción del paciente en cuanto a la estructura organizacional del servicio que se brinda en las Clínicas de la Facultad de Odontología se investigaron las siguientes dimensiones: acceso y rapidez; comodidades y señal. También, las siguientes dimensiones: Competencia técnica; relaciones interpersonales; continuidad; seguridad.

Para determinar la percepción del paciente en cuanto a resultados logrados con la atención recibida en las Clínicas de la Facultad de Odontología se investigaron las dimensiones: Eficacia; eficiencia.

El método de recolección de información empleado en este estudio fue la entrevista. Los materiales que se utilizaron fueron: Cuaderno de campo, tablet; grabadora, computadora. 


\section{RESULTADOS Y DISCUSIÓN}

$\mathrm{L}$

os resultados se expresan en función de las preguntas realizadas y las respuestas frecuentes obtenidas de la opinión de los pacientes encuestados.

Tabla 1 Resumen de los resultados obtenidos

\begin{tabular}{l}
\hline Consulta hecha \\
\hline ¿Cómo llegas a las Clínicas de la \\
Facultad de Odontología? \\
\\
¿Paga el costo del tratamiento que \\
le otorga el estudiante?
\end{tabular}

\section{Resultados}

La mayoría de las personas que asisten a las Clínicas de Odontología, vienen de zonas lejanas y utilizan como medio de transporte el servicio público de microbús, tomando en algunos casos hasta dos para llegar al lugar.

le otorga el estudiante?

¿Qué le parece el costo de los tratamientos dentales?

¿Qué tiempo espera para que el estudiante le atienda?

¿Qué servicios le gustaría que existan en la sala de espera?

¿Cuándo ingresó a la Facultad le indicaron o se dio cuenta dónde debía ir?

¿El estudiante que le atiende, le explica con detalle el procedimiento del tratamiento que le tiene que hacer?

¿Cómo es la enseñanza del doctor que le da al estudiante que le atiende?
La mayoría de los pacientes no pagan los tratamientos y argumentan que son gratuitos. Solo cancelan aspectos mínimos que necesita el estudiante para poder finalizar el tratamiento como ser radiografías y materiales que se usan en la clínica de prótesis fija y prótesis removibles.

Todos los pacientes que vienen a la clínica que fueron entrevistados, señalan que los tratamientos son gratis o muy baratos.

Realizando un análisis por especialidades, se tiene que en la Clínica de Cirugía y Periodoncia la mayoría de los pacientes son captados en la puerta de la Facultad, donde el estudiante les invita a pasar por la clínica a ser atendido, por lo que la atención es inmediata y no esperan nada, al igual que en la Clínica Odontopediatría donde los pacientes son llevados por los mismos estudiantes. Por otra parte, en las Clínicas de Ortodoncia, Operatoria Dental Endodoncia y Prótesis Fija y Removible, los pacientes tienen previamente una cita marcada, donde el tiempo de espera supera a los treinta minutos.

La mayoría de los pacientes consideran que las salas de espera no son cómodas y desconocen los servicios que tienen, como el wifi y menos saben cómo acceder, ya que la manera de acceder al wifi en las clínicas es muy complicada. Los televisores que existen en la sala de espera no están encendidos o la señal no funciona. Algunos pacientes solicitan que en la sala de espera, haya revistas y periódicos, mismos que actualmente no existen.

Por las respuestas se aprecia que no existe señalética en los ambientes de la entrada a la Facultad, la sala de espera ni pasillos, por lo que cuando el paciente ingresa no sabe dónde dirigirse ni a quien preguntar.

En las respuestas se observa que el estudiante explica con sus palabras detalladamente que tratamiento le hará al paciente, todos refirieron recibir información.

El paciente no conoce mucho al docente a cargo de la clínica sino más bien al interno, pocas veces el paciente nombro al docente y lo describe de enseñanza detallada. 


\begin{tabular}{l} 
Consulta hecha \\
\hline ¿El doctor que está a cargo de la \\
clínica supervisa constantemente \\
al estudiante? \\
¿El doctor revisa el tratamiento \\
cuando lo finaliza el estudiante?
\end{tabular}

¿La atención que recibe se realiza a la hora en la cual le han dado cita a usted?

¿Qué tan amable es el estudiante que le atiende?

¿El doctor que le enseña al estudiante que le atiende, es amable con usted?

¿Tienen paciencia cuando le atienden o cuando le explican algo?

¿Alguna vez ha sufrido algún maltrato por parte de las personas que lo atienden?

¿Qué siente cuando espera a que le atiendan? ¿Aburrimiento? ¿Ansiedad? ¿O tiene paciencia?

¿Le gustaría volver a las clínicas para poder recibir otro tratamiento?

¿Qué le parece la limpieza de las clínicas?

¿Cree que el estudiante que le atiende, le atiende con material limpio?

¿Está satisfecho con el tratamiento dental que le hicieron?

\section{Resultados}

Los encuestados señalan que no siempre esta cuando el estudiante lo necesita, y se observa que como hay demasiados estudiantes en la clínica el docente a cargo, por lo que no puede observar a todos los pacientes.

En las respuestas se aprecia que el docente siempre revisa cuando el estudiante termina el tratamiento, todos los pacientes lo confirman con sus respuestas.

En general, los pacientes consideran que los estudiantes brindan una atención puntual, principalmente en la clínica de cirugía y periodoncia donde la mayoría de los pacientes son captados en la puerta de la Facultad. Sin embargo, en algunos casos consideran que los estudiantes no siempre son puntuales en las citas.

Siempre es muy amable con el paciente y le trata con mucho respeto. Confirmado por las respuestas

De la misma manera, el paciente considera que el docente encargado de la clínica también es muy amable con él.

Los pacientes entrevistados afirman que los estudiantes y el docente demostraron paciencia durante la atención. Sus respuestas reafirman que el docente es muy respetuoso.

El paciente mediante sus afirma que la amabilidad y el respeto siempre está presente.

La respuesta del paciente es muy subjetiva al decir si se siente cansado, aburrido o con ansiedad también depende mucho del tiempo de espera. Los pacientes jóvenes se aburren más fácilmente que los pacientes de la tercera edad que esperan para la clínica de prótesis removible.

Todos desean volver a las clínicas por otros Tratamientos.

Como se señala en las respuestas, a todos los pacientes les parecen limpios los ambientes de las clínicas, sin embargo, presentan opinión negativa que sobre los baños argumentan que desprenden un mal olor.

Los pacientes no están seguros que el material que utiliza el estudiante sea limpio, la mayoría les parece que sí, aunque presentan dudas.

La mayoría de los pacientes, con algunas excepciones, están satisfechos con el tratamiento dental recibido. 


\begin{tabular}{|c|c|}
\hline Consulta hecha & Resultados \\
\hline $\begin{array}{l}\text { ¿Ha sentido mejoría con el } \\
\text { tratamiento dental que le han } \\
\text { realizado? }\end{array}$ & $\begin{array}{l}\text { En las respuestas se observa que gran parte de los } \\
\text { pacientes sintieron mejoría después del tratamiento, con } \\
\text { excepción de los pacientes de la clínica de endodoncia de } \\
\text { la tarde donde al paciente le sigue doliendo al morder o } \\
\text { alguna vez espontáneamente. }\end{array}$ \\
\hline
\end{tabular}

¿Le han realizado el tratamiento que usted esperaba o deseaba?

¿Le hacen rápido el tratamiento o le hacen volver más vec.es?
En las respuestas se observa que en su mayoría a los pacientes si les realizaron el tratamiento que ellos esperaban.
Los pacientes señalaron en la Clínica Cirugía- Periodoncia que les hicieron rápido el tratamiento $\mathrm{y}$ no necesitan volver; en la Clínica de Operatoria y Endodoncia les hacen volver más veces, lo mismo pasa en la Clínica de Ortodoncia, Pediatría, Prótesis Fija y Prótesis Removible.

\section{Discusión}

El proceso de atención en salud es el conjunto de intervenciones o procedimientos realizados, o mandados realizar, por el médico para cuidar a los pacientes y subsanar sus problemas de salud. Un proceso de atención debe centrarse en el paciente, y dar respuestas efectivas a las necesidades, valores y preferencias de los pacientes (5).

Otro concepto pertenece a la OMS plantea que la atención en salud es: "Conjuntos de acciones directas y especificas destinadas a poner al alcance del mayor número posible de individuos, su familia y la comunidad los recursos de la promoción, prevención, recuperación y rehabilitación de la salud" (OMS) (6).

Un nuevo concepto de lo que significa la atención en salud, indica: "La aplicación de todos los conocimientos médicos relevantes, la investigación básica y aplicada para aumentar estos conocimientos y hacerlos más precisos, los servicios de todo el personal médico y conexo, instituciones y laboratorios, los recursos del gobierno, agencias voluntarias y sociales y la responsabilidad de cada individuo en la cooperación" (7).

Un concepto de atención médica indica: La atención médica es el tipo de medicina que practican los líderes reconocidos de la profesión médica en un cierto periodo del desarrollo social, cultural y profesional de una determinada comunidad o grupo de población (8).Con relación a Calidad de Atención en Salud, Donabedian (1), propuso en 1980 una definición de calidad asistencial que ha llegado a ser clásica y que formulaba que la: "Calidad de la atención es aquella que se espera que pueda proporcionar al usuario el máximo y más completo bienestar después de valorar el balance de ganancias y pérdidas".

La calidad de la asistencia puede medirse como el grado de cumplimiento de un objetivo y por tanto depende de cómo se define éste. El concepto de calidad varía en función de quién lo utiliza y en consecuencia la idea de calidad será distinta cuando la aplica la administración, la gerencia del centro, que cuando el término es aplicado por los profesionales y aún por los usuarios (9).

En 1991 la Organización Mundial de la Salud afirmaba que: "Una atención sanitaria de alta calidad es la que identifica las necesidades de salud (educativas, preventivas, curativas y de mantenimiento) de los individuos o de la población, de una forma total y precisa y destina los recursos (humanos y otros), de forma oportuna y tan efectiva como el 
estado actual del conocimiento lo permite" (10).

Quizá la definición más sencilla de la calidad haya sido inspirada por el trabajo de W. Edwards Deming (11), "hacer lo correcto en la manera correcta". En los campos de salud, esto significa ofrecer una gama de servicios que sean seguros y eficaces y que cubran las necesidades y los deseos del cliente.

Desde una perspectiva de salud pública, la calidad significa ofrecer los mayores beneficios a la salud, con la menor cantidad de riesgos a la salud, a la mayor cantidad de personas, dados los recursos disponibles. Además, una buena calidad puede significar cubrir los estándares mínimos para una atención adecuada o alcanzar altos estándares de excelencia.

También se refiere al "Conjunto de arreglos y actividades que tienen por fin salvaguardar, mantener y promover la calidad de atención de la salud". Avedis Donabedian (12). Lo define como "Un proceso sistemático de cubrir la brecha entre el desempeño real y los resultados ideales".

La Administración de Calidad Total (ACT) a los servicios de salud es un enfoque organizacional integrado, para satisfacer las necesidades y expectativas del paciente, lo que comprende la participación de la administración y del personal para mejorar los procesos y servicios, mediante el uso de técnicas cuantitativas e instrumentos analíticos. De acuerdo con esta filosofía, la mejora continua de la calidad es un programa independiente de supervisión destinado a asegurar que existan todas las actividades de calidad necesarias y que sean de una eficacia óptima (13).

Ahora bien, los orígenes del concepto de calidad asistencial conducen a la preocupación por la calidad de la asistencia sanitaria que es tan antigua como la profesión médica. En los antecedentes más remotos es habitual hacer alusión al Código de Hammurabi (14) (con multas en caso de malos resultados) y al Juramento Hipocrático (15). La esencia de este compromiso ético pasa por buscar lo mejor para el paciente.

Los orígenes recientes de la preocupación por la calidad en el ámbito de la salud están asociados a la reducción de la variabilidad innecesaria en los procesos de diagnóstico, tratamiento y cuidados (16).

\section{CONCLUSIÓN}

$\mathrm{E}$ $n$ cuanto a la percepción del paciente respecto a la estructura organizacional del servicio que se brinda en las Clínicas de Odontología, la mayoría de los pacientes acuden de zonas lejanas, no pagan los tratamientos $\mathrm{y}$ argumentan que son gratuitos; esto denota que el paciente desconoce el costo real del tratamiento que le realizan. La atención en la Clínica de Cirugía y Periodoncia es inmediata al igual que en la Clínica Odontopediatría, lo que no sucede en la demás Clínicas, donde el tiempo de espera supera a los treinta minutos. Los pacientes consideran que las salas de espera no son cómodas, los televisores no están encendidos o la señal no funciona $y$ solicitan que haya revistas y periódicos. No existe señalética en los ambientes de la entrada a la Facultad, la sala de espera ni pasillos que permita orientar al paciente en su ingreso

En cuanto a la percepción del paciente respecto a los procesos de atención que le brinda el estudiante, el personal docente y administrativo en las Clínicas de Odontología, se concluye que el paciente percibe que el estudiante explica con sus palabras detalladamente el tratamiento a realizar, brindando una atención puntual, principalmente en la clínica de cirugía, además que la amabilidad y el respeto 
siempre están presente tanto por parte del estudiante y el docente. Sin embargo, el paciente no conoce mucho al docente a cargo de la clínica sino más bien al interno ya que el docente tiene a cargo a demasiados estudiantes en la clínica por lo que no puede observar a todos los pacientes. Los pacientes refieren sentir cansancio, aburrimiento y ansiedad, dependiendo del tiempo de espera, más, argumentan desear volver a las clínicas por otros tratamientos. Los ambientes de las clínicas les parecen limpios, sin embargo, llama la atención la opinión negativa que tienen sobre los baños.

En su mayoría los pacientes, están satisfechos con el tratamiento dental recibido y sintieron mejoría después del mismo, con excepción de algunos pacientes de endodoncia que argumentan seguir sintiendo dolor al morder.

La percepción del paciente en relación a la calidad del servicio y atención odontológica que se le brinda en las Clínicas de la Facultad de Odontología, es buena.

Se recomienda mejorar la relación cuantitativa docente- estudiante con el fin de optimizar el seguimiento que realiza el docente a los trabajos de los estudiantes y establecer mecanismos de seguimiento a la eficacia de los tratamientos realizados en las Clínicas Odontológicas.

- Conflicto de intereses. Ninguno declarado por la autora.

- Financiación. Ninguna declarada por la autora.

- Agradecimiento: Ninguno manifestado por la autora.

- Investigación realizada considerando los tratados bioéticos.

\section{REFERENCIAS BIBLIOGRÁFICAS}

1. Donabedian A. Explorations in quality assessment and monitoring. The definition of quality and approaches to its assessment, the criteria and standards of quality, 1982; III

2. INASES. Guía técnica de gestión de calidad para servicios de odontología de los entes gestores del sistema boliviano de la seguridad social a corto plazo. 2008

3. Morales-García. Percepción y consumo de servicios de atención odontológica en el municipio de Comalcalco, Tabasco. Salud en tabasco. 2014; 20,2

4. Saturno P. "modelos de gestion de la calidad aplicados a la atención primaria opina la universdad" Universidad de Murcia Página web Consultado en mayo 2017. Disponible en

https://cursos.campusvirtualsp.org/p luginfile.php/2346/mod_resource/co ntent/1/

Modulo_4/Lecturas_Complementarias /Semana_3_M4.S3_L_OPCIONAL_01.m odelos_gest_y_aten_primaria pdf

5. Gubio P. Estudio Descriptivo Sobre El Nivel De Satisfacción De Pacientes Que Acuden Al Servicio De Odontología Del Centro De Salud Pomasqui Del Distrito 17d03 De La Parroquia Pomasqui Del Distrito Metropolitano De Quito Durante El Período Enero A Marzo Del 2014. Tesis - Post Grado. Quito: Universidad Central Del Ecuador, Odontología; 2014

6. Organización Mundial de la Salud. La calidad de los servicios de salud. Ed. OMS. 1991. Consultado el 17 de mayo de 2018. Página web disponible en: https://apps.who.int/iris/bitstream/ handle/10665/66983/WHO_NMH_MS D_MDP_01_1_sapdf.jsessionid=4CDF3 24D24182622EA68CF3AE049BA4A?s equence $=1$

7. Rutstein S. Effects of preceding birth intervals on neonatal, infant and underfive year's mortality and nutritional status in developing contries: evidence from the Demographic and Health Surveys. Int I Gynaecol Obstet. 2005; 89 (1):7-24

8. Singer C. Salud y comunidad. La perspectiva de los cuidadores de salud Nueva York, Estados Unidos. ED. Independent. 2005 
9. Sanchón M. "Salud pública y atención primaria de salud". Consultado el 17 de mayo de 2018. Disponible en: https://ocw.unican.es/pluginfile.php/ 965/course/section/1088/2.4_los_sis temas_sanitarios.pdf

10. Organización Mundial de la Salud. La calidad de los servicios de salud. Ed. OMS 1991. Consultado el 17 de mayo de 2018. Disponible en: https://apps.who.int/iris/bitstream/ handle/10665/66983/WHO_NMH_MS D_MDP_01_1_sapdf.jsessionid=4CDF3 24D24182622EA68CF3AE049BA4A?s equence $=1$

11. Deming E. Calidad, productividad y competitividad. Nueva York. Segunda Edición. Ed. McGraw Hill. 2007

12. Donabedian A. The methods and findings of quality assessment and monitoring: An illstrate analysis. Ann Arbor: Health Administration Press, 1985.III
13. Berwick. How WE Can Rescue Health Care by Inproving It, Jossey-Bass Publishehers, Sâo Francisco, 2014

14. Franco G. Las leyes de Hammurabi. Consultado el 20 junio 2018. Disponible enhttps://rcsdigital.homestead.com/fi les/Vol_VI_Nm_3_1962/Franco.pdf

15. Robín E. Juramento Hipocrático Plasticidad y Restauración Neurológica. 2003; 2;(2):131-132

16. Saturno P. "modelos de gestion de la calidad aplicados a la atención primaria opina la universidad" Universidad de Murcia. Consultado en mayo 2018. Disponible en https://cursos.campusvirtualsp.org/p luginfile.php/2346/mod_resource/co ntent/1/

Modulo_4/Lecturas_Complementarias /Semana_3_M4.S3_L_OPCIONAL_01.m odelos_gest_y_aten_ primaria pdf 